\title{
Article \\ Credit-Default Swaps Trading in CCPs
}

\author{
Freddy Rojas Cama ${ }^{1, \dagger}$, (D)
}

1 Bay Atlantic University; Bahçeşehir University; frcama@bau.edu

* Correspondence: 1510 H street NW Floor 4th; Tel.: +1-202-740-1313

check for updates

Citation: Rojas Cama, Freddy. Credit-Default Swaps Trading in CCPs. Journal Not Specified 2021, $1,0$. https://doi.org/

\begin{abstract}
This paper shows the influence of CCP's collateralization on the pricing of the Credit Default Swaps (CDS). A narrowly variant in the way the CDS seller decides over the resources in the settlement comes with a substantial change on the elements that determine the price of the CDS.
\end{abstract}

Keywords: price discovery; financial economics; clearing; credit default swaps; collateralization; OTC; risk premium; CCP.

\section{Introduction}

This paper analyses how collateralization affects credit default swaps (CDS) premium as well as discusses further implications of above practices on counterparty risk and welfare. By setting up a population of risk-averse agents, the effects of embedding collateralization are quantitively explored over the [1]'s mechanism whose measure of risk premium and recovery rate are endogenously determined. This paper compares two contracts that differs in how the seller's resources are earmarked in the resolution of the contract. The clearinghouse or central counter-party (CCP), by operating as an escrow-account agent holding collateral, observes the risky positions of participants but its knowledge of the probability of their occurrence is limited. This remaining of the paper is structured as follows. In section (2) formally presents the model, functions to maximize and participants. Section (3) shows the results stemming from a calibrated model. Finally, section (5) presents conclusions. Appendix contains figures and the program solutions.

\section{Materials and Methods}

The CCP can set a collateral policy or initial margin requirement affecting the amount of insurance $(m)$. The model's setup follows closely to [1]'s work concerning the moral hazard in the insurer side. In particular, no clearing policies are discussed in that foregoing model. The need of protection against default risk makes a party to enter into a contract of derivatives. The seller of this derivative promises to compensate or pay out in the occurrence of a specific event that produces a loss to the buyer. This buyer agrees to make periodical payments to the seller of the protection. The provision of insurance is embedded in a model of two periods $(0,1)$ where a seller provides insurance to buyers, both risk-averse. The seller maximizes an expected mean-variance function defined as in [1], i.e $E[u(x)] \equiv E(x-f(x))+f(E(x))$. Being $f(x)=\frac{\gamma}{2} x^{2}$ and $\gamma$ defines the degree of risk-aversion attitude of the participants.

The matching amid participants in the insurance market is completely random. Only sellers can default and this assumption is made by construction since the amount of premium is payable up front. In the case of defaulting, sellers undergo a non-pecuniary penalty or deadweight loss $(\varepsilon)$ as a function of the short positions defaulted upon. Formally, the timing and actions of participants in the insurance market are presented as follows;

$\mathrm{T}=0 \quad$ The CCP sets the collateral requirement. A group of buyers and one seller -randomly matched- observe the above requirement and decide to trade a CDS. They sign a contract specifying amount of insurance $(m)$, premium $(q)$, and the collateral $(\kappa)$, last two in terms of per unit of insurance. The premium is pay up front.

$\mathrm{T}=1 \quad$ Seller gets the endowment $\omega_{s}$ if credit even happens and also gets a gross return $\bar{\theta}$ from a portfolio. With probability $\lambda$ the reference entity or bond defaults; seller makes a choice 
from set $\imath=\{N D, D\}$ where $N D$ and $D$ stand for non-default and default respectively. Uncertainty is resolved; CCP verifies the positions and the settlement and payoffs to each participants are made. Otherwise, with probability $1-\lambda$ no transfers are made.

The next program (P1) is solved in order to show the effect of collateralization on the CDS premium. The seller defaults if the recovery rate $(R)$ and the fraction of the notional -which is collateralized- is less than 1, i.e. $R+\kappa<1$. In this program the seller sets the restriction $\omega_{s} \geq R m$. In a non-default equilibrium, this restriction is binding because i) CCP observes $\omega_{s}$ at $T=1$, and ii) it must cover the portion of the notional which is not collateralized, i.e. $\omega_{s}=(1-\kappa) m$. The seller knows that will surely receive $\omega_{s}$ if the credit event happens, the CCP does know the amount of the endowment but its occurrence. Regarding the latter, The Korea Exchange (KRX) episode ${ }^{1}$ has proven that the CCP can substantially misjudge the creditworthiness of its clearing participants or be inadequately incentivized to manage risk properly (see [4]). Taking into account, in a general view, the possibility of incomplete or limited information, the setup in the model assumes that CCP underestimates the seller's financial solvency.

It must be noticed that clearinghouses choose collateral as a fraction of the size of notional, thus they setup the collateral policy. Both seller and buyer maximize their profit or utility functions choosing the level of insurance. Additionally, the market clear condition is required in equilibrium. Functions $\Pi^{B}, \Pi_{D}^{S}$ and $\Pi_{N D}^{S}$ are as follows;

$$
\begin{gathered}
\Pi_{D}^{S}=\bar{\theta}+\omega_{s, 0}+q m-v \kappa\left(m-\omega_{s}\right)+\lambda\left(\omega_{s}-(R+\kappa+\varepsilon) m\right)-\frac{\gamma_{s}}{2} \lambda(1-\lambda)\left[\omega_{s}-(\varepsilon+\kappa+R) m\right]^{2}-c_{p} \\
\Pi_{N D}^{S}=\bar{\theta}+\omega_{s, 0}+q m-v \kappa\left(m-\omega_{s}\right)+\lambda\left(\omega_{s}-m\right)-\frac{\gamma_{s}}{2} \lambda(1-\lambda)\left[\omega_{s}-m\right]^{2}-c_{p} \\
\Pi^{B}=\omega_{b, 0}-q m+(1-\lambda) \omega_{b}+\lambda(R+\kappa) m-\frac{\gamma_{b}}{2} \lambda(1-\lambda)\left[\omega_{b}-(R+\kappa) m\right]^{2}
\end{gathered}
$$

The program is as follows,

$$
\begin{aligned}
& \mathscr{L}=\max _{\kappa}\left\{\max _{m^{s}}\left(\Pi_{N D}^{S} \mathbb{1}_{\{R+\kappa=1\}}+\Pi_{D}^{S} \mathbb{1}_{\{R+\kappa<1\}}\right)^{\sigma} \times \max _{m^{b}} \Pi^{B}\right\} \\
& \text { subject to } \quad m \equiv m^{s}=(N+1) m^{b} \\
& \alpha q m+\bar{\theta}-\kappa m-v \kappa\left(m-\omega_{s}\right) \geq c_{p} \\
& q=(\Delta \lambda+\lambda)(R+\kappa) \\
& 1 \geq R+\kappa \\
& \omega_{s} \geq R m \\
& 1 \geq \kappa \geq 0
\end{aligned}
$$

In above program, the CCP -from the point of view of a regulator or benevolent social plannermaximizes the product of seller and buyers' utility functions, the parameter $\sigma$ measures the relative importance of the seller's utility when a policy is implemented. The parameter $v$ is the cost of posting collateral and it is a percentage of the netting and collateralized exposure between the seller and the CCP. The first restriction is the market clearing condition. The second restriction is the participation constraint. The third restriction is the CDS premium; this expression is determined by the seller and taken as given by the clearinghouse, a key component of the contract price is the risk premium $(\Delta \lambda)$. The fourth restriction is an expression -given by the terms of the contract- that limits the sum of recovery rate and the collateral to be greater than one. The restriction $\omega_{s} \geq R m$ is very significant since establishes both the seller's limited liability and the risky position that is observable to the CCP at $\mathrm{T}=1$ (see appendix A). Finally, restriction on the range of $\kappa$ that allows for corner solutions is formally shown in above program. 
The optimal insurance levels for sellers and buyers are as follows,

$$
\begin{aligned}
m_{D}^{s}= & \frac{q-\lambda \varepsilon-v \kappa-\lambda(R+\kappa)+\psi_{1}(\alpha q-\eta \kappa)-\psi_{2} R}{\gamma_{s} \lambda(1-\lambda)(\varepsilon+\kappa)(R+\kappa+\varepsilon)} \\
m_{N D}^{s}= & \omega_{s} \\
& m^{b}=\frac{1}{R+\kappa}\left[\frac{-q+\lambda(R+\kappa)}{\gamma_{b} \lambda(1-\lambda)(R+\kappa)}+\omega_{b}\right]
\end{aligned}
$$

$\psi$ are Lagrange multipliers associated to above inequalities shown in the program (see appendix for details).

The second resolution of the settlement $(\mathrm{P} 2)$ permits a constraint $\omega_{s} \geq(R+\kappa) m$ which replaces $\omega_{s} \geq R m$ in the above program (P1). This restriction guarantees that the endowment covers also the amount of collateral posted in $T=0$. In both programs, the constrains are binding in the solution. Also, under this policy in both programs the buyer's budget constraint remains slack.

A benchmark allocation that represents an efficient result amid participants in this fictional financial economy is formally stated below;

Benchmark or First Best. [1] states that if the total amount of insurance and endowments were observable, a planner could impose a pricing rule on $q\left(m, \omega_{s}, \Delta \lambda\right)$ to be $(i)[\Delta \lambda+\lambda]$ whenever there is no default, and (ii) $[\Delta \lambda+\lambda] \frac{\omega_{s}}{m}$ when there is default. When substituting the price rule into CDS seller's maximization, the incentives to default vanishes. Buyers continue to purchase insurance in a competitive way taking the price as given. The result will be no supply of insurance in equilibrium beyond the non-defaulting insurance level (see [1] for details).

\section{Results}

The model proposed in the previous section is used to show the impact of the CCP policy (collateralization) on the premium. The parameter $N$ refers to extra buyers, this controls the leverage position of each seller. After the great recession, these positions are believed to be small due to regulation; thus $N=1$.

Figure (1) shows the baseline parameterization. The probability of occurrence of the credit event $(\lambda)$ is fixed to $5 \%$ and it represents last figures from rating agencies' data ${ }^{2}$. The risk aversion parameter for the buyer $\left(\gamma_{b}\right)$ is conservative and equal to 0.7 according to own calculations and supported by figures reported in $[9]^{3}$. On the other side of the contract, literature usually sets a low risk parameter value for the seller $\left(\gamma_{s}\right)$, for instance [4] pins the parameter to zero. In the exercise the value for $\gamma_{s}$ is assumed to be low and fixed to 0.2 ; the aforementioned value is somehow arbitrary but enough low though to catch a significance response of the recovery rate. The parameter $\alpha$ is fixed to $20 \%$ and it represents the fraction of premium available to face any call from clearinghouse; it certainly has impact on the size of seller's participation constraint and it allows the available resources to peter out in a controlled way. The possible loss or endowment $\left(\omega_{b}\right)$ in the last period for the buyer is equal to 7 ; since it is assumed a leverage of $40 \%$ for each seller, then the number of buyers per each contract is equal to 2 i.e. $N=1$. The average return of portfolio $(\bar{\theta})$ is equal to 3 whereas fixed costs of participation in clearing $\left(c_{p}\right)$ is equal to 1 . The endowment received in last period for seller $\left(\omega_{s}\right)$ is equal to 10 .

2 [5] stated that according to Fitch data reported in [6] the high-yield bond default rate is around 4.7\% as last figure for 2017. S\&P rating services (see [7]) reported $3.9 \%$ of default rate in 2016 and also calculated a pessimistic scenario of 5.2\% for 2017 . Although default rate is historically low, there are vulnerable companies that are more likely to slide into default; mostly those companies' operational fields are commodities related such as oil, gas and mining steel. Furthermore, [8] shows that global speculative-grade default rate declined quickly immediately after the great recession; thus, sectors as banking, non-bank finance, transportation and utilities had default rates of less of $1 \%$. The sectors with highest rates were only media and retail with $3.6 \%$ and $3.3 \%$ respectively.

3 [9] reckons a CDS spread equivalent to the expected loss times a risk adjustment which provides a definition of price of default risk a.k.a risk aversion measure. The aforementioned is equivalent to the expression $\frac{\Delta \lambda}{\lambda}$; thus, assuming a value of 1.42 (average value reported in [9]) and a leverage of 1.4 (ratio of exposure to equity), the parameter $\gamma$ is equal to 0.7 . 
Table 1: Baseline parameterization

\begin{tabular}{lrl}
\hline Parameters & Value & Definition \\
\hline$\lambda$ & 0.05 & Probability of the event \\
$\sigma$ & 0.25 & Weight on the Utility function \\
$\gamma_{b}$ & 0.70 & Risk aversion parameter of buyer \\
$\gamma_{s}$ & 0.20 & Risk aversion parameter of seller \\
$\omega_{s}$ & 10.00 & CDS seller's endowment at $\mathrm{t}=1$ \\
$\omega_{s, 0}$ & 0.00 & CDS seller's endowment at $\mathrm{t}=0$ \\
$\bar{\theta}$ & 3.00 & CDS seller's Portfolio return \\
$c_{p}$ & 1.00 & Participation costs or clearing fee in the CCP \\
$\omega_{b}$ & 7.00 & CDS buyer's loss \\
$\omega_{b, 0}$ & 0.00 & CDS buyer's endowment at $\mathrm{t}=0$ \\
$\alpha$ & 0.20 & Limited liability parameter \\
$v$ & 0.02 & Opportunity cost of collateral \\
$N$ & 1.00 & Number of extra buyers \\
\hline
\end{tabular}

The values of insurance, recovery rate, CDS premium and risk premium as part of the solution of the model are shown in figures (1), (2), (3) and (4). The results are discussed in the next section.
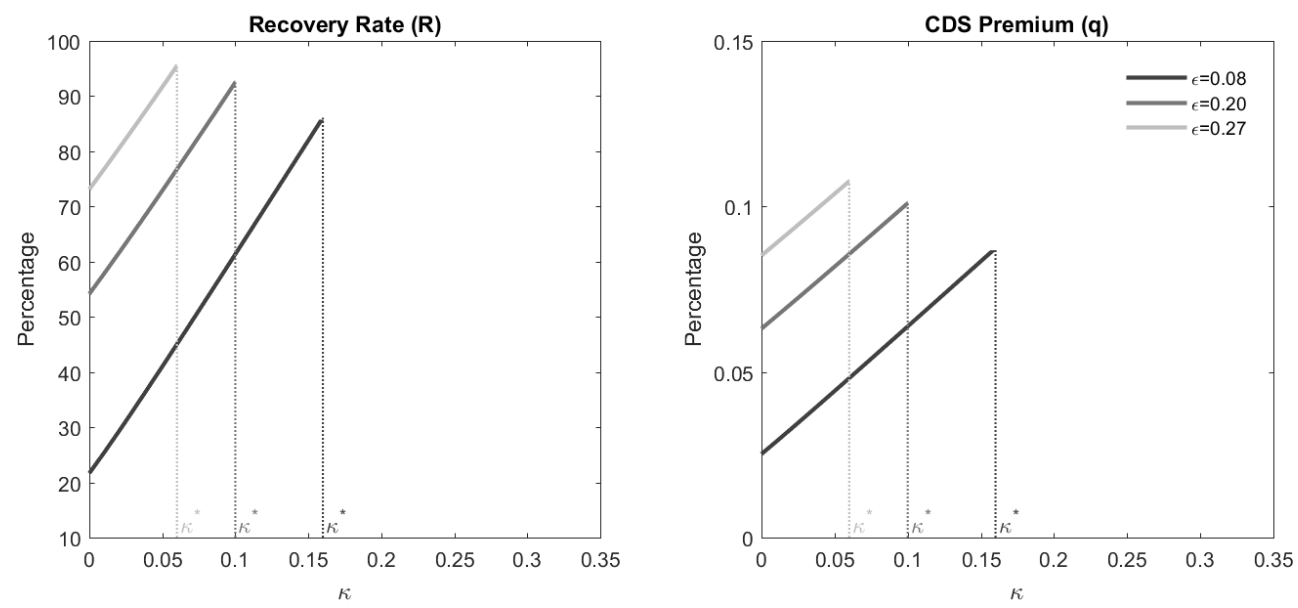

Figure 1. Recovery rate and CDS premium for P1; non-defaulting contracts when $\varepsilon<\varepsilon^{*}$
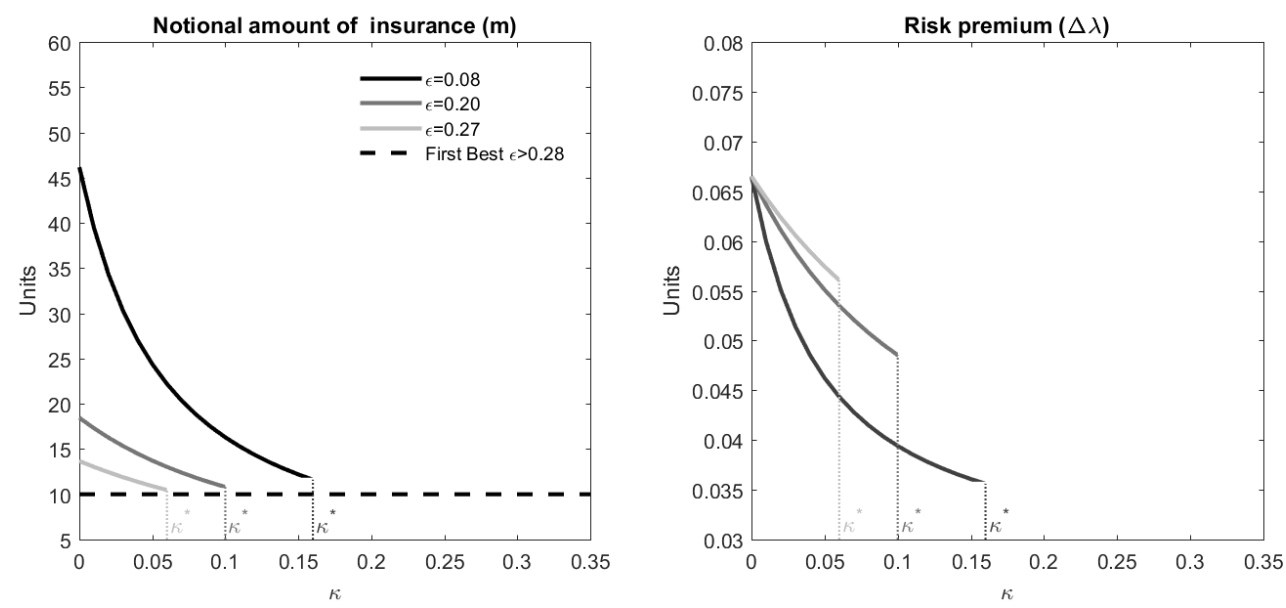

Figure 2. Notional and risk premium for $\mathrm{P} 1$ when $\varepsilon<\varepsilon^{*}$ 

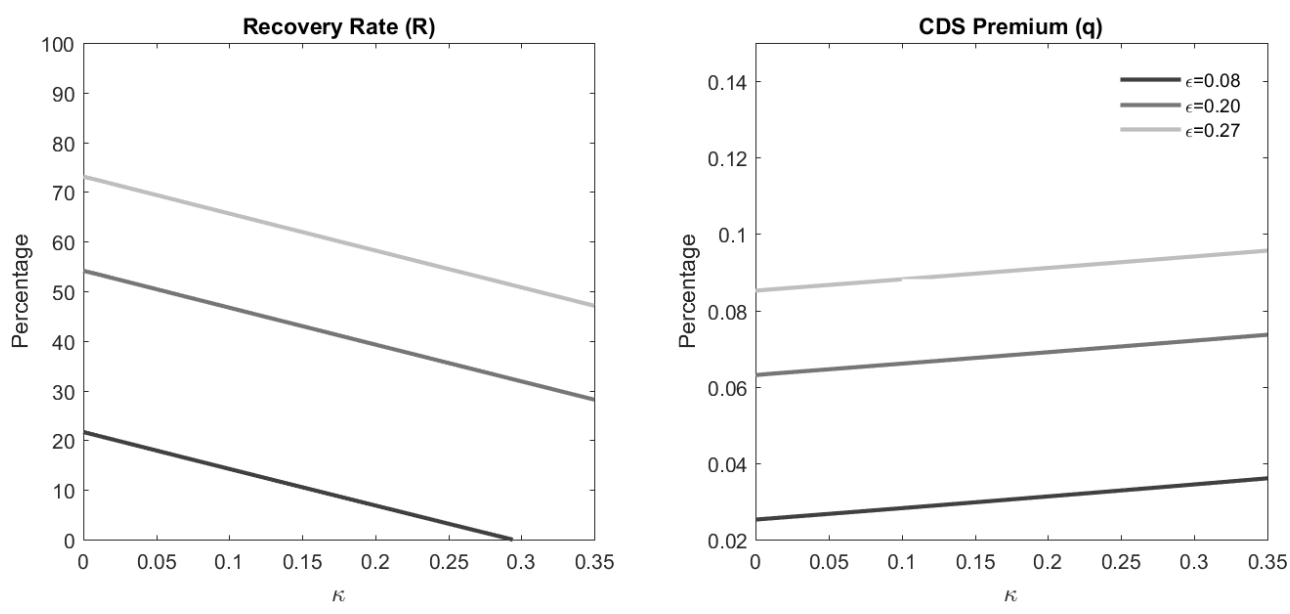

Figure 3. Recovery rate and CDS premium for the program (P2); defaulting contracts when $\varepsilon<\varepsilon^{*}$
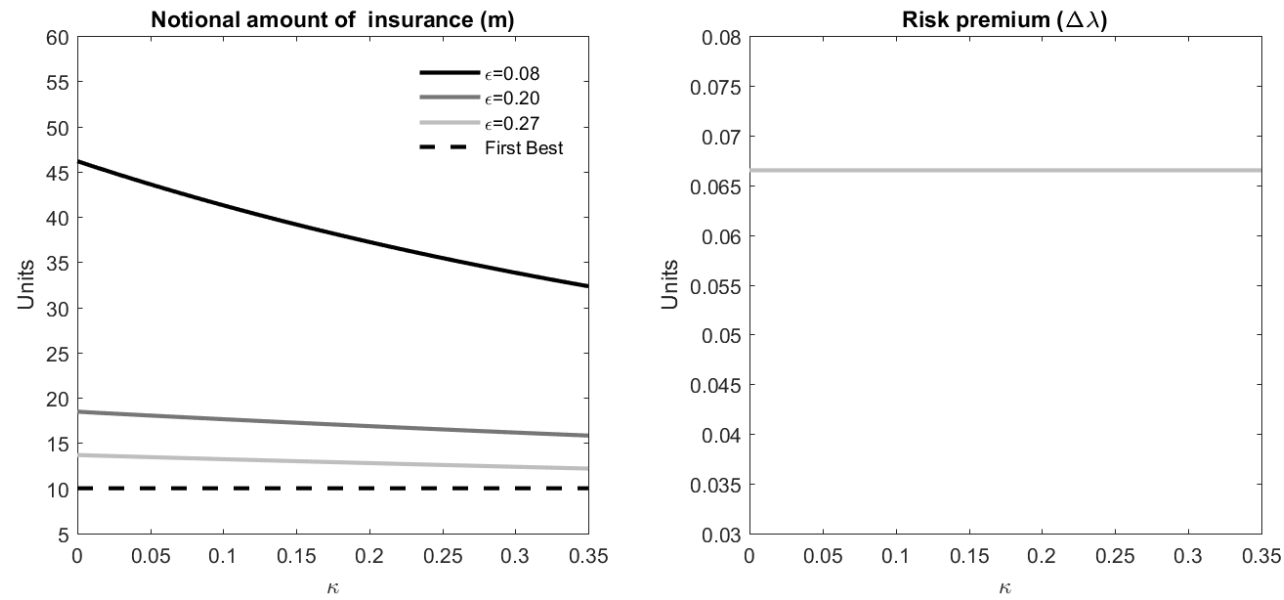

Figure 4. Notional and risk premium for the program (P2) when $\varepsilon<\varepsilon^{*}$

\section{Discussion}

Figure (1) shows the recovery rate and CDS premium affected by the collateral policy. In particular above figure shows three cases -three sizes of $\varepsilon$ - where a incentive contract (see [10]) can be implemented; the incentive contract shows that for a large level of deadweight loss, there is some collateral level $\kappa^{*}$ that makes the seller do not default. The existence of $\kappa^{*}$ is a possibility since the participation constraint must remain slack. Depending of the level of $\varepsilon$, there are different collateral requirements implemented by an incentive contract. Here two points to remark: $i$ ) collateral requirement makes both recovery rate $(R)$ and premium $(q)$ increase; and $i i)$ Ratio $(R+\kappa) / q$ is increasing, this is a consequence of a decreasing risk premium $(\Delta \lambda)$. The contracts related to aforementioned sizes of $\varepsilon$ are not longer under default, a result that holds in equilibrium; a result only possible when $\varepsilon$ is large enough and the participation constraint is slack enough.

Figure (2) shows that CDS notional amount $(m)$ is affected by the clearinghouse's collateral policy. $R+\kappa$ and $q$ increases due to collateralization, also those requirements negatively affect the demand of insurance $\left(m^{b}\right)$.

Also, above figure shows, as long as recovery rate is less and close to $1-\kappa$, the amount of insurance reaches a value slightly above the first best. Higher $\varepsilon$ means higher recovery rates and therefore clearinghouse will require less collateral and a non-defaulting outcome will hold in equilibrium. The figure (2) also shows the risk premium as a function of collateral levels. The risk premium declines as collateral increases. The aforementioned effect on risk premium is produced by the restriction $\omega_{s}=R m$ that holds as solution to the problem solved by the clearinghouse. The resolution for the settlement in (P2) guarantees the restriction $\omega_{s}=(R+\kappa) m$ to hold as solution to the seller's problem. The restriction makes a difference in terms of the risk premium effects on the 
premium; the size of the risk premium is constant as in [1]. For the above settlement, the figure (3) shows that $\mathrm{R}$ decreases and the premium increases. The figure (4) shows that the amount of notional is larger in comparison to the solution provided in the program (P1).

In terms of welfare (see appendix $\mathrm{C}$ ) the total welfare is increasing in the size of collateral taken by the CCP for the program (P1). In the program (P2) the total welfare decreases for a small value of deadweight losses. The foregoing may suggest that trading in a OTC may be beneficial for at least the seller.

\section{Conclusions}

The [1]'s original model is modified to include a clearinghouse in the insurance market that imperfectly observes the size of participants' positions and collect collateral from the seller. The main finding of this paper is that regulation has a significant effect on recovery rate and risk premium, key components of the CDS premium, and it differs on how the seller decides over the resolution of the settlement. In this scenario the CCP operates as an escrow-account agent holding collateral.

If the seller decides that the amount of recovery is equal to the position on a third-party i.e. $\omega_{s}=R m^{s}$, the CCP is likely to offer a non-defaulting contract. If the seller decides that the collateral amount is a fraction of that position i.e. $\omega_{s}=(R+\kappa) m^{s}$, the CCP is less likely to offer a non-defaulting contract. In both cases the CDS premium increases; however, the level of that price will differ for a specific dead-weight loss. Also, in the last model, for a particular low value of parameter $\varepsilon$, the CCP collateralization policy does not achieve any gain in welfare utils. In that case, in order to avoid the clearing fees, the CDS contract might be traded in OTC markets rather than in a CCP.

The results in this paper are similar to empirical ones shown in [11] suggesting that clearing practices causes an increase in CDS spreads through its impact on the value of the contract. The novel approach in this paper lays out a theoretical model that allows to parse other new and significant channels. Thus, this paper finds a force that pushes down the premium in a specific clearing-policy environment where fully-insured contracts spread significantly, the model offers a channel where risk premium is affected by clearing policies. This paper with an assumption of no competition (random matches $)^{4}$ shows that there is a channel where a component of the CDS premium declines. However, in the proposed setup, a different channel -via recovery rate- prevails and makes the premium to increase ultimately.

Funding: "This research received no external funding"

Conflicts of Interest: "The author declares no conflict of interest."

Abbreviations
The following abbreviations are used in this manuscript:
MDPI Multidisciplinary Digital Publishing Institute
DOAJ Directory of open access journals
TLA Three letter acronym
LD Linear dichroism

\section{Appendix A Netting through novation}

The clearinghouse stands among the buyer (B), seller (S) and a third party (TP). An arrow indicates "exposure to ..."

\footnotetext{
4 See [12]'s work which shows that price competition is a necessary and sufficient condition for a low premium under mutualization of contracts with high counterparty risk.
} 


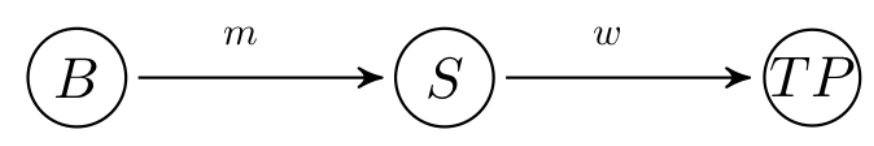

Figure A1. OTC

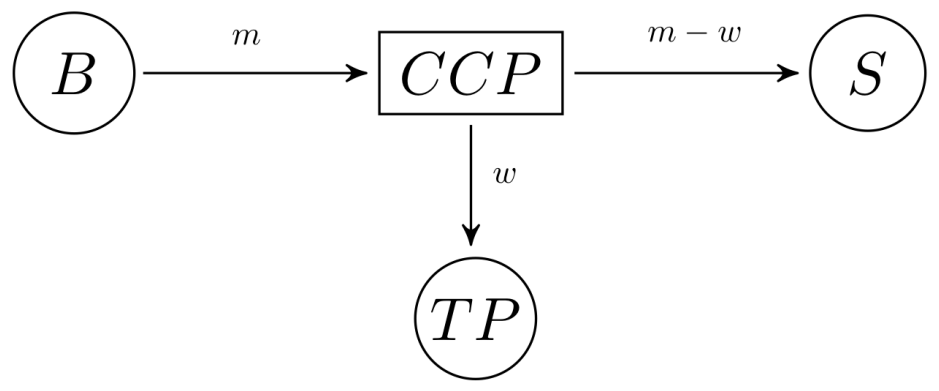

Figure A2. Clearing

\section{Appendix B Solution for programs}

In order to solve the program (P1 and P2) a numerical method is proposed. Since the function is not differentiable at $R+\kappa=1$, the solution is found in the following steps:

1. I setup a search grid for the collateral size in the limits $\kappa \in[0, \bar{\kappa}]$.

2. If the set $\left\{\kappa \mid \bar{\theta}+\alpha q(\varepsilon) m(\varepsilon) \geq \kappa m(\varepsilon)+v \kappa\left(m(\varepsilon)-\omega_{s}\right)-c_{p}\right\}$ is not empty for a small enough $\varepsilon$ then there is a candidate $\kappa^{c}$.

3. If restrictions in program (P1) are fulfilled then $\kappa^{c}$ is a solution and premium associated to this level of collateral is the premium under collateralization. Otherwise, $\kappa^{*}=0$ and the premium is identical under OTC negotiation.

The Lagrange for the seller (in a default scenario) is:

$$
\begin{aligned}
L_{D}^{s}= & \bar{\theta}+\omega_{s, 0}+q m-v \kappa\left(m-\omega_{s}\right)+\lambda\left(\omega_{s}-(R+\kappa+\varepsilon) m\right)-\frac{\gamma_{s}}{2} \lambda(1-\lambda)\left[\omega_{s}-(\varepsilon+\kappa+R) m\right]^{2}-c_{p} \\
& +\psi_{1}\left(\alpha q m+\bar{\theta}-\kappa m-v \kappa\left(m-\omega_{s}\right)-c_{p}\right)+\psi_{2}\left(\omega_{s}-R m\right)+\psi_{3}(1-R-\kappa)+\psi_{4} \kappa+\psi_{5}(1-\kappa)
\end{aligned}
$$

The [13] and [14] conditions are invoked for the solution. The proposed solution entails to consider that $\psi_{4}$ and $\psi_{5}$ are equal to zero (interior solution). The participation constraint is slack; therefore $\psi_{1}$ equal to zero. The constraint $\omega_{s}-R m^{s}$ is binding and the $\psi_{2}$ equal to zero thus fulfilling the condition that it must be non-negative. In the default scenario $R+\kappa<1$ thus $\psi_{3}$ equal to zero. In the program (P1) -see MATLAB code attached to the paper-, there are four restrictions: two market clear conditions for the default and non-default case, the participation constraint times $\psi_{1}$ and the $\frac{\omega_{s}}{R}=(N+1) m^{b}$ which combined with the market clear condition for the default case gives the constraint $\frac{\omega_{s}}{R}=m^{s}$. The buyer maximizes $\Pi^{B}$ with no restriction.

The solution for the program (P2) assumes that $\omega_{s}-(R+\kappa) m^{s}$ is binding and the values for all respective $\psi$ 's are the same as in the case of program (P1). In the program (P2) -see MATLAB code attached to the paper- the foregoing restriction is equivalent to the clearing market condition for the case of non-default. 


\section{Appendix C Welfare}
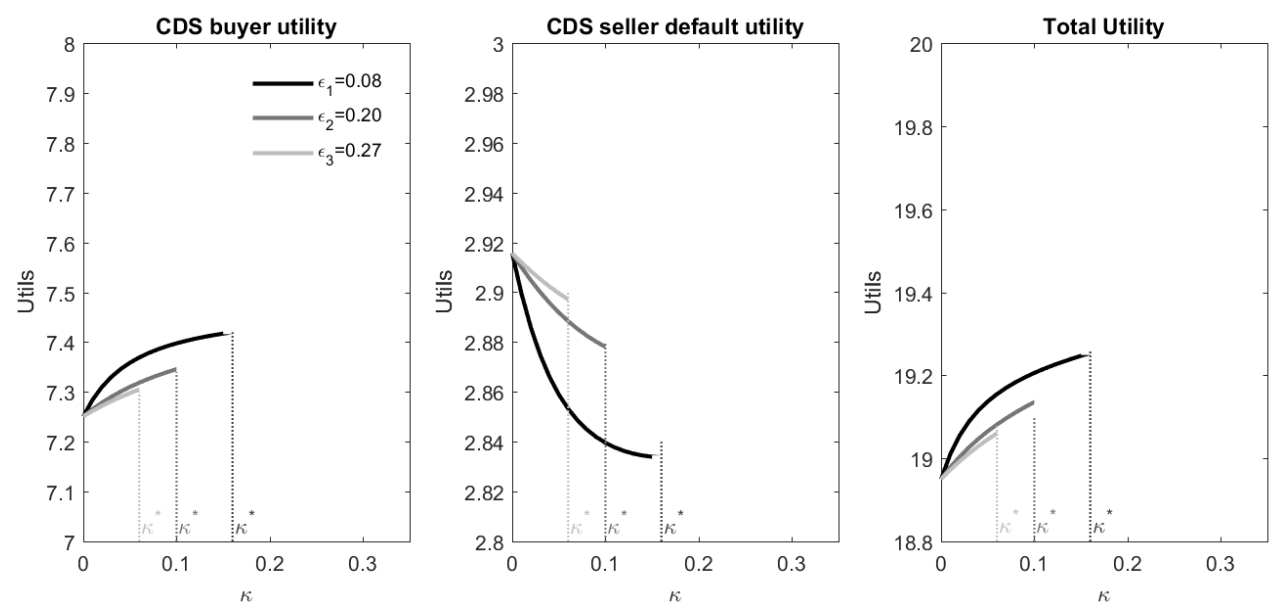

Figure A3. Welfare in the program P1
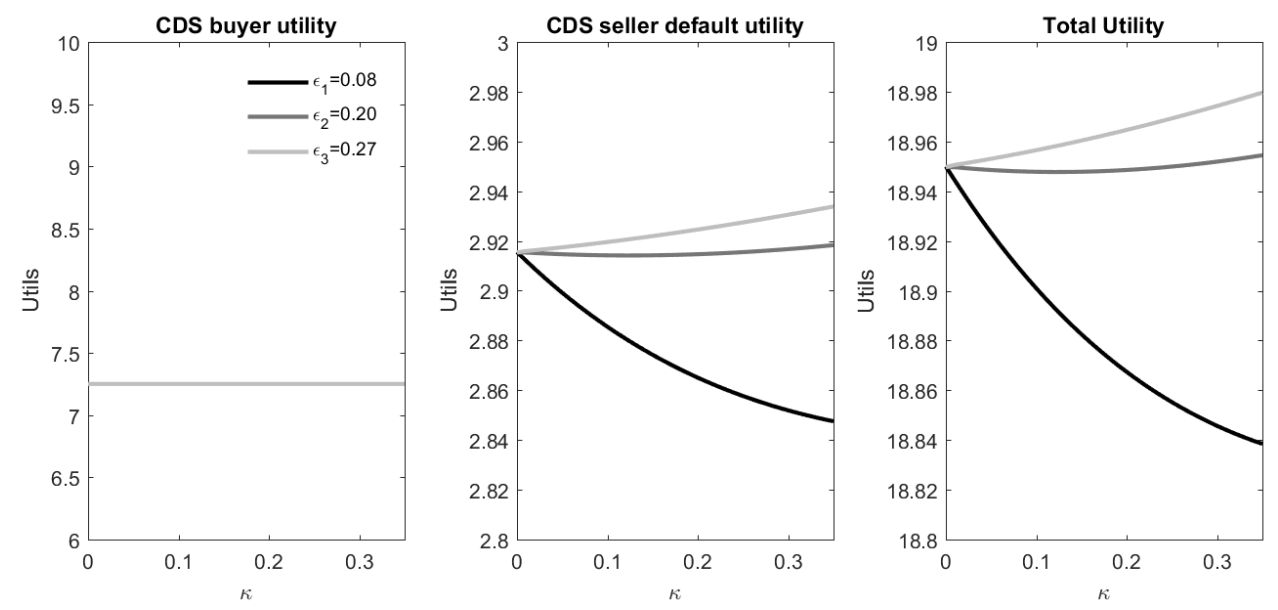

Figure A4. Welfare in the program 2

\section{References}

1. Acharya, V.; Bisin, A. Counterparty risk externality: Centralized versus over-the-counter markets. Journal of Economic Theory 2014, $149,153-182$.

2. Vaghela, V. Korea trading error prompts global clearing house review, (2014. [Online; posted 4th-March-2014 in Risk.Net].

3. Grant, J. Banks Launch Clearing Review after Korean Broker Default, (2014. [Online; posted 7th-March-2014 in Financial Times].

4. Huang, W. Central Counterparty Capitalization and Misaligned Incentives. Bank for International Settlements Working Paper Series 2019, 767.

5. Cama, F.A.R. The Effect of Mutualization and Collateralization on Credit Default Swaps Premium. PhD thesis chapter II 2016.

6. Kenny, T. Default Rates and Bonds. www.thebalance.com/what-is-the-default-rate-416917, (2019.

7. Vazza, D. CNBC Interview high-yield default rises MON, APR 4 20163:52 PM EDT., (2016.

8. Moodys. Corporate Default and Recovery Rates, 1920-2010. Special Report, Moodys Investors Service 2011.

9. Amato, J. Risk Aversion and Risk Premia in the CDS market. BIS Quarterly Review, December 20052005.

10. Koeppl, T. The Limits of Counterparty Clearing: Collusive Moral Hazard and Market Liquidity. Queen's Economic Department Working Paper 2013.

11. Loon, Y.C.; Zhong, Z. The impact of central clearing on counterparty risk, liquidity, and trading: Evidence from the credit default swap market. Working Paper 2014.

12. Stephens, E.; Thompson, J.R. CDS as Insurance: Leaky Lifeboats in Stormy Seas. Working paper 2011. 
13. Kuhn, H.W.; Tucker, A.W. Non-linear programming. Proceedings of 2nd Berkeley Symposium. Berkeley: University of California Press. 1951.

14. Karush, W. Minima of Functions of Several Variables with Inequalities as Side Constraints. Mimeograph, Dept. of Mathematics, Univ. of Chicago, Chicago, Illinois 1939. 